\title{
ORIGINAL ARTICLE High-dose methotrexate-based immuno-chemotherapy for elderly primary CNS lymphoma patients (PRIMAIN study)
}

K Fritsch ${ }^{1,26}$, B Kasenda ${ }^{2,3,26}$, E Schorb ${ }^{1}, \mathrm{P} \mathrm{Hau}^{4}$, J Bloehdorn ${ }^{5}$, R Möhle ${ }^{6}, \mathrm{~S} \mathrm{Löw}^{7}, \mathrm{M} \mathrm{Binder}^{8}$, J Atta ${ }^{9}, \mathrm{U} \mathrm{Keller}^{10}$, H-H Wolf ${ }^{11}$, SW Krause ${ }^{12}$, $\mathrm{G} \mathrm{He}^{13}{ }^{13}$ R Naumann ${ }^{14}$, S Sasse ${ }^{15}, \mathrm{C} \mathrm{Hirt}^{16}, \mathrm{M} \mathrm{Lamprecht}^{17}$, U Martens ${ }^{18}$, A Morgner $^{19}$, J Panse ${ }^{20}, \mathrm{~N} \mathrm{Frickhofen}^{21}, \mathrm{~A} \mathrm{Röth}^{22}, \mathrm{C} \mathrm{Hader}^{23}$, $\mathrm{M}$ Deckert $^{24}$, H Fricker $^{1}$, G Ihorst ${ }^{25}$, J Finke ${ }^{1,26}$ and G Illerhaus ${ }^{1,2,26}$

To investigate immuno-chemotherapy for elderly immuno-competent patients ( $\geqslant 65$ years) with newly diagnosed primary central nervous system lymphoma, we conducted a multicentre single-arm trial. One cycle consisted of rituximab $\left(375 \mathrm{mg} / \mathrm{m}^{2}\right.$, days $1,15,29)$, high-dose methotrexate $\left(3 \mathrm{~g} / \mathrm{m}^{2}\right.$ days $\left.2,16,30\right)$, procarbazine $\left(60 \mathrm{mg} / \mathrm{m}^{2}\right.$ days $\left.2-11\right)$ and lomustine $\left(110 \mathrm{mg} / \mathrm{m}^{2}\right.$, day 2)-R-MPL protocol. Owing to infectious complications, we omitted lomustine during the study and consecutive patients were treated with the R-MP protocol. Three cycles were scheduled and repeated on day 43. Subsequently, patients commenced 4 weekly maintenance treatment with procarbazine (100 mg for 5 days). Primary end point was complete remission (CR) after 3 cycles. We included 107 patients (69 treated with R-MPL and 38 with R-MP). In all, 38/107 patients achieved CR (35.5\%) and 15 (14.0\%) achieved partial remission. R-MP was associated with a lower CR rate (31.6\%) compared with R-MPL (37.7\%), but respective 2-year progression-free survival (All 37.3\%; R-MP 34.9\%; R-MPL 38.8\%) and overall survival (All 47.0\%; R-MP 47.7\%; R-MPL 46.0\%) rates were similar. R-MP was associated with less $\geqslant$ grade 3 toxicities compared with R-MPL (71.1\% vs $87.0 \%)$. $\mathrm{R}-\mathrm{MP}$ is more feasible while still associated with similar efficacy compared with R-MPL and warrants further improvement in future studies.

Leukemia (2017) 31, 846-852; doi:10.1038/leu.2016.334

\section{INTRODUCTION}

Primary central nervous system lymphoma (PCNSL) is a malignant diffuse large B-cell lymphoma confined to the CNS. PCNSL is an orphan disease accounting for $<5 \%$ of all primary brain tumours and $4-6 \%$ of all extra-nodal lymphomas. ${ }^{1}$ Rituximab and highdose methotrexate (HD-MTX) in combination with HD-cytarabine (HD-AraC) is the backbone of current treatment. ${ }^{2,3}$ Patients $>60$ years account for $50 \%$ of all PCNSL cases, and although they are able to tolerate systemic chemotherapy elderly patients are more seriously affected by iatrogenic toxicity, especially leukoencephalopathy and neurological side effects following whole-brain radiotherapy. ${ }^{4}$ Therefore, they represent a unique and vulnerable treatment subgroup in which whole-brain radiotherapy is avoided whenever possible., ${ }^{5,6}$ A recent meta-analysis revealed that evidence for the optimal treatment approach in elderly patients is still limited; however, a combination of HD-MTX and oral alkylating agents was associated with favourable outcomes. ${ }^{7}$ We previously reported on a prospective single-centre study with rituximab, HD-MTX, procarbazine and lomustine (R-MPL) in 28 elderly PCNSL patients. ${ }^{8}$ Complete remissions (CRs) were observed in 64\%; 3-year progression-free survival (PFS) was 31\%. Based on that pilot study, we initiated a multicentre single-arm phase II trial to test the R-MPL protocol plus procarbazine maintenance in elderly patients $\geqslant 65$ years with newly diagnosed PCNSL.

\section{PATIENTS AND METHODS}

\section{Study design}

This was a prospective single-arm phase II trial conducted at 20 centres in Germany registered at clinicaltrials.gov (NCT 00989352).

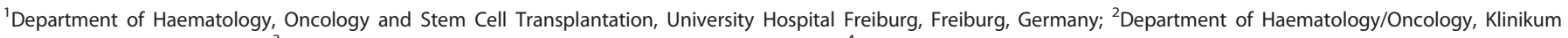

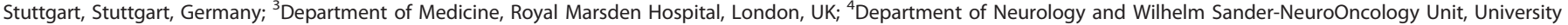

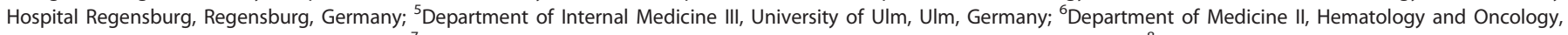

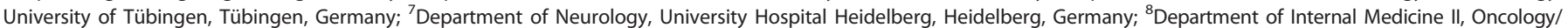

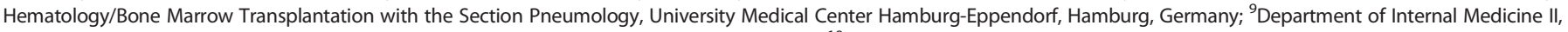

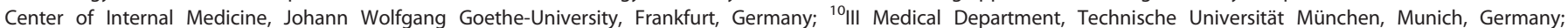

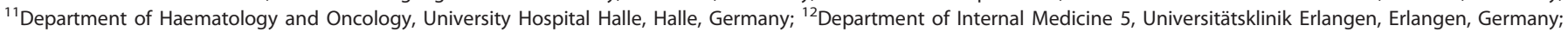

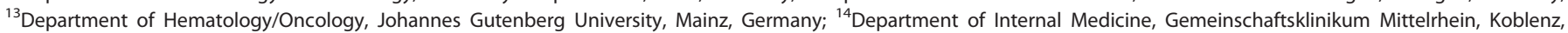

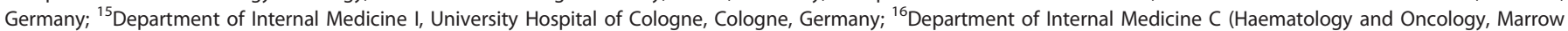

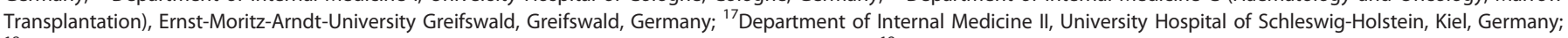

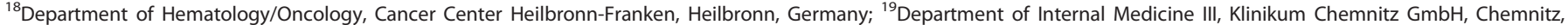

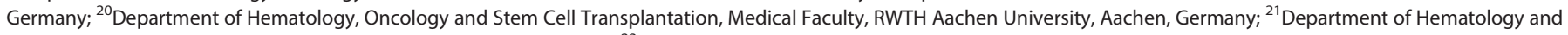

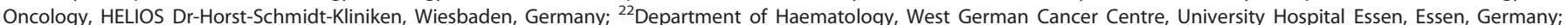

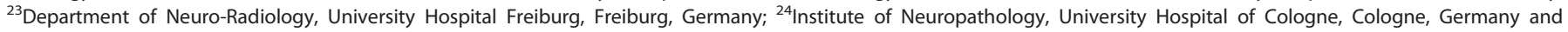

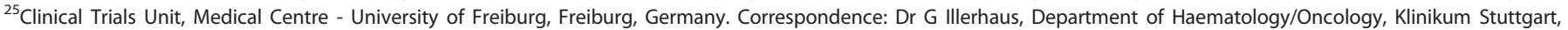
Kriegsbergstrasse 60, Stuttgart 70174, Germany.

E-mail: g.illerhaus@klinikum-stuttgart.de

${ }^{26}$ These authors contributed equally to this work.

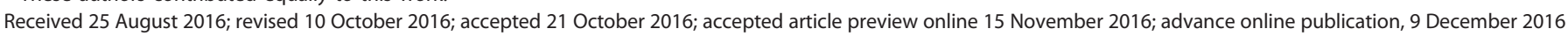


Eligibility criteria

Immuno-competent patients with newly diagnosed PCNSL (proven on histology) according to the World Health Organization criteria aged $\geqslant 65$ years were eligible irrespective of clinical performance status. Diagnosis was established by neuropathological analysis of tumour samples using conventional histology and immunohistochemistry with CD20, BCL6, MUM1 and MIB-1 staining. Patients required baseline staging, including gadolinium enhanced brain magnetic resonance imaging, slit-lamp examination and lumbar puncture, if not contraindicated. To rule out systemic involvement, computed tomographic body scans and bone marrow examinations were mandatory. Exclusion criteria were HIV seropositivity, inadequate bone marrow function (defined as neutrophils $<1.5 \times 10^{9} /$ l, platelets $\left.<100 \times 10^{9} / \mathrm{l}\right)$, cardiac ejection fraction $<50 \%$, creatinine clearance $<50 \mathrm{ml} / \mathrm{min}$ and severe non-compensated pulmonary or liver disease with bilirubin $>2 \mathrm{mg} / \mathrm{dl}$.

\section{Treatment}

One cycle of the initial protocol (R-MPL) consisted of: rituximab $375 \mathrm{mg} / \mathrm{m}^{2}$ infused in $90 \mathrm{~min}$ on day -6 (only at the beginning of treatment), days 1 , 15 and 29; HD-MTX $3 \mathrm{~g} / \mathrm{m}^{2}$ over $4 \mathrm{~h}$ on days 2, 16 and 30; lomustine $110 \mathrm{mg} / \mathrm{m}^{2}$ orally on day 2 , and procarbazine $60 \mathrm{mg} / \mathrm{m}^{2}$ orally on days $2-11$. Initial steroid treatment was allowed and tapered once HD-MTX infusion started. Cycles were repeated every 42 days with 3 cycles planned in total. Maintenance treatment with procarbazine $100 \mathrm{mg}$ for 5 days $(6$ cycles repeated on day 29) was started on day 43 of the last R-MPL cycle. Maintenance treatment was also allowed if not all 3 R-MPL cycles were applied. After protocol amendment, lomustine was omitted, but the other immuno-chemotherapy components remained unchanged resulting in the R-MP protocol-mandatory pneumocystis jiroveci pneumonia prophylaxis was also added. From June 2012 onwards, all patients were treated with R-MP.

All patients or guardians provided written informed consent. The study conformed to the tenets of the Declaration of Helsinki and approved by the local ethics committee at Freiburg University and the ethics committees at participating centres.

\section{Response evaluation}

We used magnetic resonance imaging scans after every cycle to assess response as recommended. ${ }^{9}$ Lumbar puncture and slit-lamp examination was repeated after each cycle if positive at initial staging or if a meningeal or ocular relapse was suspected clinically. Patients with $\mathrm{CR}$, partial response $(\mathrm{PR})$ or stable disease with clinical improvement were allowed to continue on protocol at each time point of evaluation. After completion of treatment, disease was assessed every 3 months within the first year, every 6 months until the fifth year and yearly thereafter. Toxicity was graded according to the Common Toxicity Criteria (CTC, version 3.0).

\section{End points}

The primary end point was the CR rate measured after 3 cycles of R-MPL. Secondary end points included toxicity, neurotoxicity as evaluated by the mini-mental status test (MMST), quality of life (QoL) using the European Organization for Research and Treatment of Cancer QoL core questionnaire (EORTC QLQ-C30 Version 3.0) ${ }^{10}$ and QLQ-BN20 for brain cancer, best response achieved during immuno-chemotherapy, PFS defined as time from the start of treatment until progression or death from any cause whichever occurred first and overall survival (OS) defined as time from the start of treatment until death from any cause.

\section{Sample size calculation and reason for amendment}

Sample size calculation was based on the primary end point using a onestage Fleming design. ${ }^{11}$ If the $C R$ rate was $\leqslant 40 \%\left(P_{0}\right)$, treatment would be considered not effective. Treatment would be considered effective if the CR rate after 3 cycles of R-MPL would at least be $60 \%\left(\mathrm{P}_{1}\right)$. Consequently, we planned a one-sided binomial test for the primary null hypothesis that the $\mathrm{CR}$ probability was $\leqslant 0.4$ at a significance level alpha $=10 \%$. The sample size was determined to provide sufficient power (95\%) to reject the null hypothesis in case the actual $C R$ probability was $\geqslant 0.6$. Based on this, we initially planned to enrol 56 patients treated according to the R-MPL protocol. Recruitment to the PRIMAIN study was very fast, therefore, the steering committee decided to continue recruitment until 112 patients, also considering that there was no alternative clinical trial for this patient population at that time. Because of several reported adverse and serious adverse events associated with treatment (mainly haematological toxicities and infections), the steering committee decided dropping lomustine without knowing effectiveness of R-MPL. This amendment was approved by the local ethics committee at Freiburg University and the ethics committees at participating centres.

\section{Statistical analysis}

The statistical analysis was conducted using SAS 9.2 (SAS Institute Inc. Cary, NC, USA) and the software R (www.r-project.org). The intention-totreat (ITT) population was defined as those registered patients fulfilling inclusion criteria and for whom treatment was initiated. For the primary end point, all patients with missing data owing to treatment discontinuation were regarded as non-responders. The per-protocol (PP) population was defined as those patients for whom no major protocol violations were known; patients discontinuing treatment owing to progression/relapse/ death or toxicity were included in the PP population. Analyses in the PP population were conducted as sensitivity analyses. Safety analyses are conducted in the ITT population. The primary end point was analysed by calculating the CR rate together with an exact two-sided $95 \%$ confidence interval $(\mathrm{Cl})$ based on the binomial distribution. Based on 107 evaluable patients, the null hypothesis could be rejected if the number of observed CRs is $\geqslant 50$. OS and PFS rates were estimated by the Kaplan-Meier method. Death owing to lymphoma (relapse/progression) rates were estimated as cumulative incidence rates with the Aalen Johanson estimator, ${ }^{12}$ considering death without prior relapse as a competing risk. For explorative purposes, the primary end point, toxicity, PFS and OS were also investigated stratified by R-MPL and R-MP. We used Cox regression modelling to investigate the prognostic impact of the following factors on OS: age, Karnofsky Performance Score ( $<70$ vs $\geqslant 70 \%$ ), localization of lymphoma (supratentorial vs infratentorial), lactate dehydrogenase (normal vs elevated), and treatment (R-MPL vs R-MP). Results from the MMST and EORTC QLQ-C30 are summarized per time point using descriptive statistics for continuous data. Comprehensive analyses of QoL outcomes will be reported elsewhere.

\section{RESULTS}

Patient characteristics

Between October 2009 and March 2013, we registered 112 patients at 20 German centres. Five patients were excluded because of systemic lymphoma involvement at registration or having a diagnosis of relapsed PCNSL. Finally, 107 eligible patients were included in the ITT population (Figure 1). Table 1 summarizes the patient characteristics; 7 (6.5\%) were $\geqslant 80$ years.

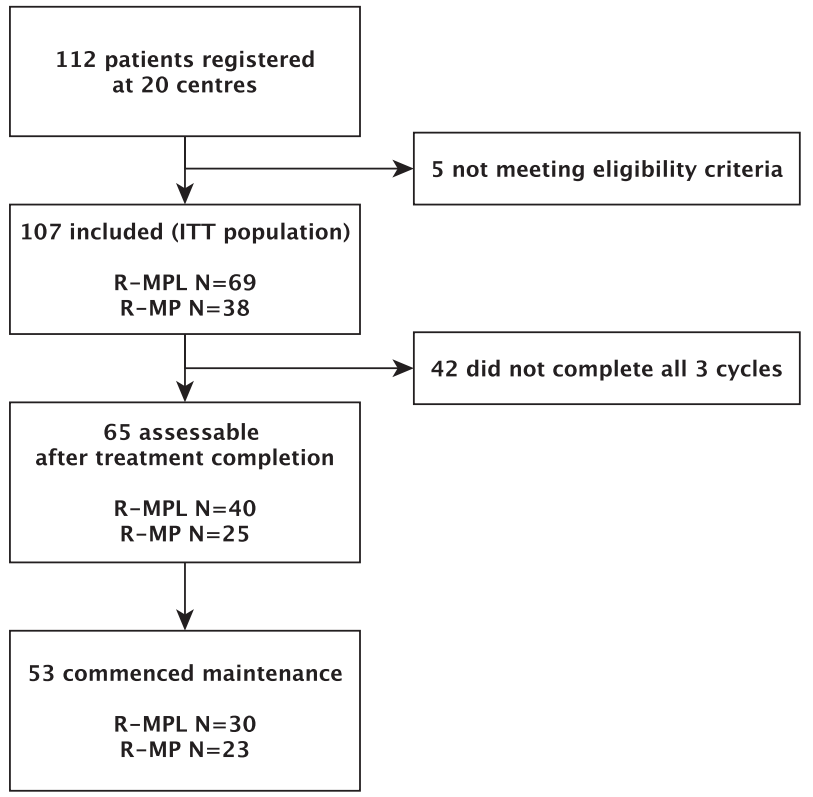

Figure 1. Patient flow in the PRIMAIN study. 
Table 1. Baseline characteristics stratified by the protocol applied

\begin{tabular}{|c|c|c|c|}
\hline Characteristics & $\begin{array}{c}R-M P L \\
(\mathrm{~N}=69)\end{array}$ & $\begin{array}{c}R-M P \\
(\mathrm{~N}=38)\end{array}$ & $\begin{array}{c}\text { All } \\
(\mathrm{N}=107)\end{array}$ \\
\hline $\begin{array}{l}\text { Median age (range), } \\
\text { years }\end{array}$ & $73(66-85)$ & $73(66-85)$ & $73(66-85)$ \\
\hline Female & $34(49.3)$ & $16(42.1)$ & $50(46.7)$ \\
\hline \multicolumn{4}{|c|}{ Karnofsky performance status ${ }^{\mathrm{a}}$} \\
\hline Median (range) & $70(30-100)$ & $60(30-100)$ & $70(30-100)$ \\
\hline$\geqslant 70 \%$ & $32(56.1)$ & $17(47.2)$ & $49(52.7)$ \\
\hline \multicolumn{4}{|l|}{ Lymphoma lesions $^{\mathrm{b}}$} \\
\hline Single & $28(41.2)$ & $13(34.2)$ & $41(38.7)$ \\
\hline Multiple & $40(58.8)$ & $25(65.8)$ & $65(61.3)$ \\
\hline \multicolumn{4}{|l|}{ Lymphoma location } \\
\hline Infratentorial & $11(16.2)$ & $10(26.3)$ & $21(19.8)$ \\
\hline Supratentorial & 50 (73.5) & $20(52.6)$ & $70(66.0)$ \\
\hline Both & $7(10.3)$ & $8(21.1)$ & $15(14.2)$ \\
\hline \multicolumn{4}{|c|}{ Serum lactate dehydrogenase $e^{c}$} \\
\hline Elevated & $30(44.8)$ & $17(45.9)$ & $47(45.2)$ \\
\hline Normal & $37(55.2)$ & $20(54.1)$ & $57(54.8)$ \\
\hline Ocular involvement & $1(1.5)$ & $0(0)$ & $1(0.9)$ \\
\hline \multicolumn{4}{|l|}{ Histology } \\
\hline DLBCL & $69(100.0)$ & $38(100.0)$ & $107(100.0)$ \\
\hline
\end{tabular}

Abbreviations: DLBCL, diffuse large B-cell lymphoma; R-MP, rituximab, methotrexate and procarbazine; R-MPL, rituximab, methotrexate, procarbazine and lomustine. Numbers are frequencies (proportions) unless

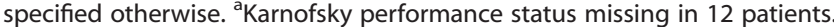
${ }^{\mathrm{b}}$ Missing in 1 patient. ${ }^{\mathrm{C}}$ Missing in 2 patients.

Feasibility and toxicity

Sixty-nine patients (65.5\%) were treated with R-MPL and 38 (35.5\%) with R-MP. In total, 53 patients (49.5\%) commenced maintenance treatment with procarbazine (R-MP 23/38 (60.5\%) vs R-MPL 30/69 (43.5\%)). Of those 53 patients, 34 (64.2\%) completed all 6 planned cycles of maintenance treatment. Eighty-seven of the 107 patients $(81.3 \%)$ experienced at least one $\geqslant$ grade 3 toxicity during R-MPL of which the most frequent were: leukopenia $(55.1 \%)$, infections $(35.5 \%)$, and anaemia (32.7\%). Patients treated with R-MP had less $\geqslant$ grade 3 toxicities (27/38 (71.1\%) vs 60/69 $(87.0 \%)$ - - particularly, severity of haematological toxicity and infections were lower (Table 2). There were 9 (8.4\%) treatmentrelated deaths: 7 with R-MPL ( 5 with sepsis which included 1 case of fatal pneumocystis jiroveci pneumonia, 1 liver failure and 1 pulmonary embolism) and 2 with R-MP ( 1 with grade 5 diarrhoea and 1 with chest sepsis).

Response after 3 cycles-primary end point

Sixty-five of the 107 (60.7\%) patients had a final scan after 3 cycles of R-MPL: 38 of the 107 patients achieved a CR $(35.5 \%$; $95 \% \mathrm{Cl}$ 26.5-45.4\%), 15 (14.0\%) had PR, and 12 (11.2\%) suffered PD or relapsed disease. The remaining 42 patients did not reach the end of treatment scan because of death $(13,12.1 \%)$, toxicity (18, $16.8 \%)$, patient wish $(4,3.7 \%)$ and other reasons $(7,6.5 \%)$-all considered as non-responders for the primary end point. Therefore, with 38 of the 107 patients in CR, the primary end point was not met. The $C R$ rate was slightly lower in patients treated with R-MP compared with R-MPL (31.6\% vs $37.7 \%$ ) (Table 3 ). Results from our sensitivity analyses (PP population) were similar with CR in 38 of the 96 patients $(39.6 \% ; 95 \% \mathrm{Cl} 29.7-50.1 \%), P R$ in 15 patients (15.6\%), and PD or relapsed disease in 12 (12.5\%).
Table 2. Toxicities during treatment

\begin{tabular}{|c|c|c|c|}
\hline Toxicity & $R-M P L(\mathrm{~N}=69)$ & $R-M P(\mathrm{~N}=38)$ & All $(\mathrm{N}=107)$ \\
\hline \multicolumn{4}{|l|}{ Leukopenia } \\
\hline Grade 1 & $3(4.4)$ & $7(19.4)$ & $10(9.6)$ \\
\hline Grade 2 & $12(17.6)$ & $8(22.2)$ & $20(19.2)$ \\
\hline Grade 3 & $32(47.1)$ & $12(33.3)$ & $44(42.3)$ \\
\hline Grade 4 & $13(19.1)$ & $2(5.6)$ & $15(14.4)$ \\
\hline \multicolumn{4}{|l|}{ Anaemia } \\
\hline Grade 1 & $6(8.8)$ & $6(16.7)$ & $12(11.5)$ \\
\hline Grade 2 & 31 (45.6) & $20(55.6)$ & $51(49.0)$ \\
\hline Grade 3 & 27 (39.7) & $6(16.7)$ & $33(31.7)$ \\
\hline Grade 4 & $2(2.9)$ & $0(0)$ & 2 (1.9) \\
\hline \multicolumn{4}{|c|}{ Thrombocytopenia } \\
\hline Grade 1 & $11(16.2)$ & $10(27.8)$ & $21(20.2)$ \\
\hline Grade 2 & $11(16.2)$ & $2(5.6)$ & $13(12.5)$ \\
\hline Grade 3 & $20(29.4)$ & $0(0)$ & $20(19.2)$ \\
\hline Grade 4 & 9 (13.2) & $2(5.6)$ & $11(10.6)$ \\
\hline \multicolumn{4}{|l|}{ Infections } \\
\hline Grade 1 & $2(2.9)$ & $3(8.3)$ & $5(4.8)$ \\
\hline Grade 2 & $12(17.6)$ & $8(22.2)$ & $20(19.2)$ \\
\hline Grade 3 & $24(35.3)$ & $8(22.2)$ & $32(30.8)$ \\
\hline Grade 4 & $3(4.4)$ & $0(0)$ & $3(2.9)$ \\
\hline \multicolumn{4}{|l|}{ Transaminases } \\
\hline Grade 1 & $27(40.3)$ & 7 (19.4) & $34(33.0)$ \\
\hline Grade 2 & $13(19.4)$ & $11(30.6)$ & $24(23.3)$ \\
\hline Grade 3 & $14(20.9)$ & $10(27.8)$ & $24(23.3)$ \\
\hline Grade 4 & $3(4.5)$ & $0(0)$ & $3(2.9)$ \\
\hline \multicolumn{4}{|c|}{ Hyperbilirubinaemia } \\
\hline Grade 1 & $13(19.1)$ & $5(13.9)$ & $18(17.3)$ \\
\hline Grade 2 & $2(2.9)$ & $0(0)$ & $2(1.9)$ \\
\hline Grade 3 & $0(0)$ & $0(0)$ & $0(0)$ \\
\hline Grade 4 & $0(0)$ & $0(0)$ & $0(0)$ \\
\hline \multicolumn{4}{|c|}{ Renal impairment (serum creatinine) } \\
\hline Grade 1 & $20(29.4)$ & $11(30.6)$ & $31(29.8)$ \\
\hline Grade 2 & $14(20.6)$ & 7 (19.4) & $21(20.2)$ \\
\hline Grade 3 & $5(7.4)$ & $1(2.8)$ & $6(5.8)$ \\
\hline Grade 4 & $0(0)$ & $0(0)$ & $0(0)$ \\
\hline \multicolumn{4}{|l|}{ Mucositis } \\
\hline Grade 1 & $11(16.4)$ & $4(11.1)$ & $15(14.6)$ \\
\hline Grade 2 & $10(14.9)$ & $4(11.1)$ & 14 (13.6) \\
\hline Grade 3 & $5(7.5)$ & $3(8.3)$ & $8(7.8)$ \\
\hline Grade 4 & $0(0)$ & 0 & $0(0)$ \\
\hline Toxic deaths & $7(10.1)$ & $2(5.3)$ & $9(8.4)$ \\
\hline
\end{tabular}

Abbreviations: R-MP, rituximab, methotrexate and procarbazine; R-MPL, rituximab, methotrexate, procarbazine and lomustine. Numbers are frequencies (proportions).

Best response achieved and survival analyses

Considering best response achieved, 45 of the 107 achieved CR (42.1\%), 34 PR (31.8\%), one SD and 2 patients suffered PD (Table 3). Twenty-five (23.4\%) patients did not have any response assessment as per protocol, because of: death $(N=10)$, toxicity $(N=9)$, lack of compliance $(N=2)$, patients' wish $(N=2)$, and progression $(N=2)$ during the first cycle. Of those 53 patients who proceeded with maintenance treatment, 37 had a CR (69.8\%), 14 PR (26.4\%) and one PD (1.9\%) before commencing procarbazine. After a median follow-up of 33.7 months, 70 (65.4\%) patients experienced relapse/progression or died (59 (55.1\%) patients died). This translated into a median PFS of 10.3 months with 1and 2 -year PFS rates of $46.3 \%$ and $37.3 \%$, respectively (Figure $2 a$ ). 
Table 3. Primary outcome (complete remission rate after 3 cycles) and secondary outcomes of effectiveness, including best-documented response achieved during whole treatment course, progression-free survival and overall survival

\begin{tabular}{|c|c|c|c|}
\hline Response after three cycles & $R-M P L(\mathrm{~N}=69)$ & $R-M P(\mathrm{~N}=38)$ & All $(\mathrm{N}=107)$ \\
\hline CR & $26(37.7,26.5-50.2)$ & $12(31.6,18.0-48.8)$ & $38(35.5,26.5-45.4)$ \\
\hline PR & $6(8.7,3.6-18.6)$ & $9(23.7,12.0-40.6)$ & $15(14.0,8.3-22.4)$ \\
\hline PD/relapse & $8(11.6,5.5-22.1)$ & $4(10.5,3.4-25.7)$ & $12(11.2,6.2-19.1)$ \\
\hline Not done & $29(42.0,30.4-54.5)$ & $13(34.2,20.1-51.4)$ & $42(42.1,32.7-52.0)$ \\
\hline \multicolumn{4}{|l|}{ Best response achieved } \\
\hline SD & $1(1.4,0.08-8.9)$ & 0 & $1(0.9,0.05-5.8)$ \\
\hline PD & $1(1.4,0.08-8.9)$ & $1(2.6,0.1-15.4)$ & $2(1.9,0.3-7.3)$ \\
\hline Not done & $17(24.6,15.4-36.7)$ & $8(21.1,10.1-37.8)$ & $25(23.4,16.0-32.7)$ \\
\hline \multicolumn{4}{|l|}{ Progression-free survival } \\
\hline Median months (95\% Cl) & $9.6(4.8-24.0)$ & $11.2(5.7-27.3)$ & $10.3(6.5-15.9)$ \\
\hline 1-year rate \% $(95 \% \mathrm{Cl})$ & $44.9(33.2-56.7)$ & $48.9(32.8-65.0)$ & $46.3(36.8-55.8)$ \\
\hline 1-year rate \% $(95 \% \mathrm{Cl})$ & $53.6(41.9-65.4)$ & $62.4(46.8-78.0)$ & $56.7(47.2-66.1)$ \\
\hline 2-year rate \% $(95 \% \mathrm{Cl})$ & $46.0(34.1-57.8)$ & $47.9(30.4-65.3)$ & $47.0(37.3-56.7)$ \\
\hline
\end{tabular}

The respective OS rates after 1 and 2 years were $56.7 \%$ and $47.0 \%$; median OS was 20.7 months (Figure 2b). Although follow-up is shorter in the R-MP group, the associated PFS and OS rates are similar compared with R-MPL (Table 3, Figures $2 \mathrm{c}$ and d). Of those seven patients aged $\geqslant 80$ years, five are still alive at latest followup. In multivariable analysis, age and all other factors had no prognostic influence on OS (Table 4).

Overall, 38 of the 59 deaths $(64.4 \%)$ were attributable to PCNSL. The cumulative incidence of death owing to PCNSL at 1 and 2 years was $23.6 \%(95 \% \mathrm{Cl} 16.7-33.2 \%)$ and $33.2 \%(95 \% \mathrm{Cl} 25.2-$ $43.9 \%$ ), respectively (Figure 2e). Cumulative incidence rates of death owing to reasons other than PCNSL were $15.0 \%$ at 6 months (95\% Cl 9.6-23.6\%), increased slightly to $19.8 \%$ at 12 months (95\% $\mathrm{Cl}$ 13.5-29.0\%) and remained the same afterwards (Figure 2f). Relapse rates during maintenance treatment and response duration of patients achieving CR during or after completion of treatment are summarized in Supplementary Figures S1 and S2.

\section{MMST and QoL}

At baseline, the mean MMST score was 22.8 (s.d. 6.6, range 0-30) but not available in 19 patients (17.8\%). After one cycle, most patients showed improved MMST scores, expressed by an intraindividual mean difference compared with baseline of +3.15 (s.d. +5.23 , range -7 to +21 ). Afterwards, the intra-individual change to baseline remained stable after the second cycle $(+3.07$, s.d. 4.77 range -4 to +17 ) but slightly improved after completion of treatment $(+3.58$, s.d. 5.33 , range -7 to +16$)$. However, owing to missing data, intra-individual changes for MMST were only calculated in $40(37.4 \%)$ patients after cycle 1, $28(26.2 \%)$ after cycle 2 and 24 (22.4\%) after completion of scheduled treatment. The summary measure Global Health Status Score (EORTC QLQ-30) over time is shown in Supplementary Figures S3 and S4.

\section{DISCUSSION}

Treatment of elderly patients with PCNSL remains a challenge. In this multicentre prospective trial, immuno-chemotherapy with
R-MPL was active but associated with significant toxicities. However, the a priori specified minimal proportion of $40 \% \mathrm{CR}$ rate after 3 cycles R-MPL was not achieved. Omitting lomustine (RMP) was associated with similar PFS and OS but less toxicity.

Our study provides a good level of evidence and results are transferable to every day clinical practice. Thanks to the efforts of all participating sites, we recruited $>100$ elderly patients in $<4$ years, which demonstrates that prospective studies in this rare disease, especially in the vulnerable subgroup of elderly patients, are feasible. We included patients aged $>65$ years irrespective of clinical performance status and had no restriction on upper age limit, truly mirroring the daily practice situation.

Our study has several limitations: Although our assumptions for sample size calculation were based on our previous single-centre pilot study, ${ }^{8}$ we could not observe the minimal expected $\mathrm{CR}$ rate of $40 \%$ after 3 cycles of R-MPL. Also, when considering bestachieved response achieved, the previously observed $C R$ rate of $64 \%{ }^{8}$ could not be repeated in this multicentre study. This is partly explained by the unexpected high proportion (40\%) of patients who did not reach the end of treatment scan after 3 cycles because of toxicities, progression or death and who were conservatively considered as non-responders in the prespecified analysis plan. However, when considering PFS, results are almost identical with a 3 -year PFS of $31 \%$ in the preceding pilot study ${ }^{8}$ and $33 \%$ in the present study. Although CR is a common end point in phase II studies, ${ }^{13}$ it may not be the appropriate primary end point in future comparative studies for elderly patients; in such an aggressive disease, PFS or even OS should be considered. Our study was not designed to compare R-MP with R-MPL and also less patients were treated with R-MP and their follow-up was shorter; therefore, any inference regarding comparative effectiveness should be considered exploratory.

Based on a recent systematic review, ${ }^{7}$ only few prospective multicentre studies focussing on elderly patients with PCNSL have been reported, ${ }^{14-20}$ which all used HD-MTX-based chemotherapy. However, no standard protocol has yet been defined and there was no study that used rituximab. In terms of patients recruited, 
a

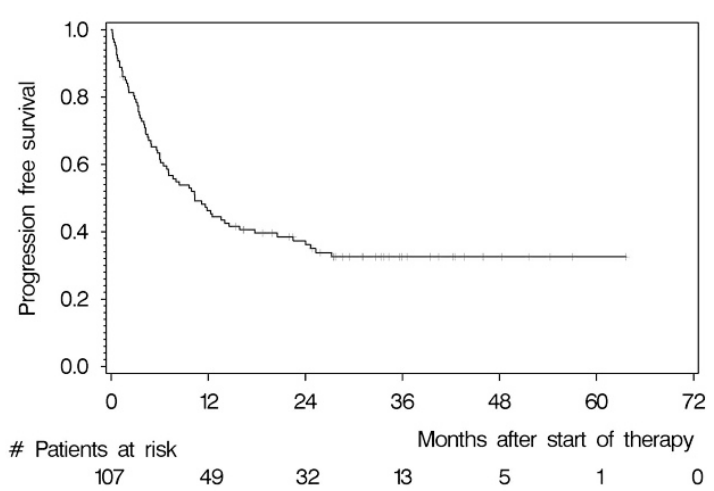

c

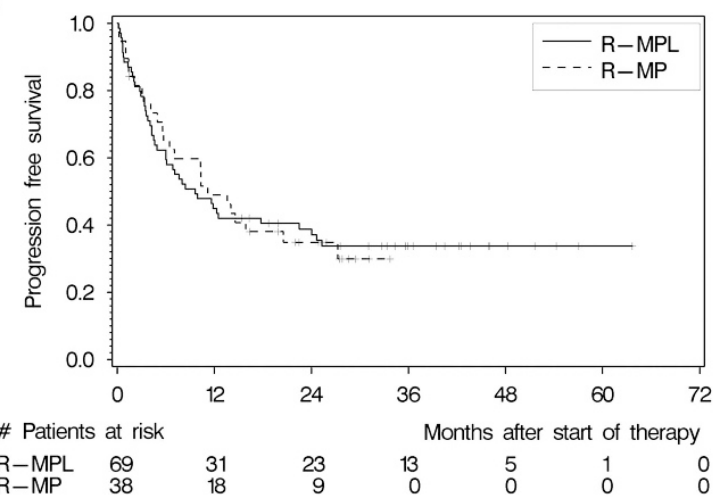

e

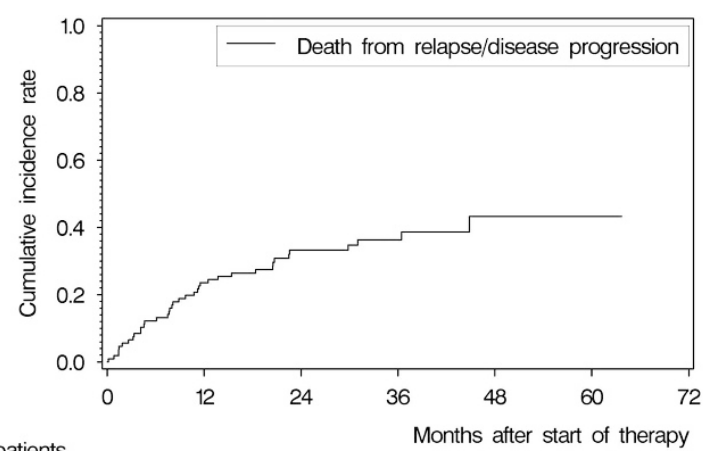

b

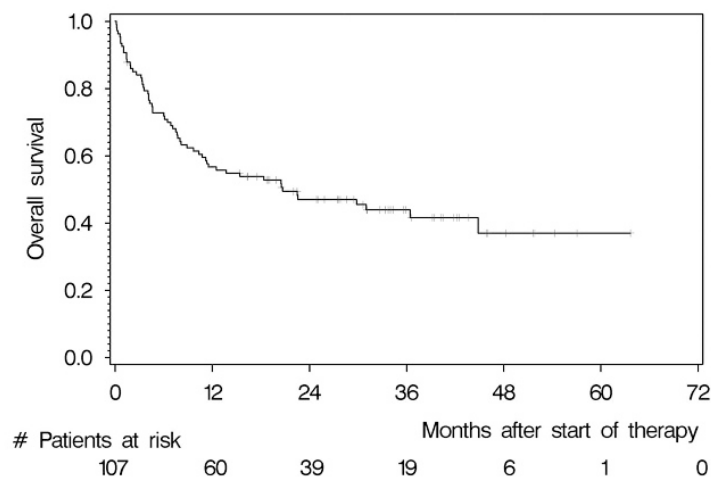

d

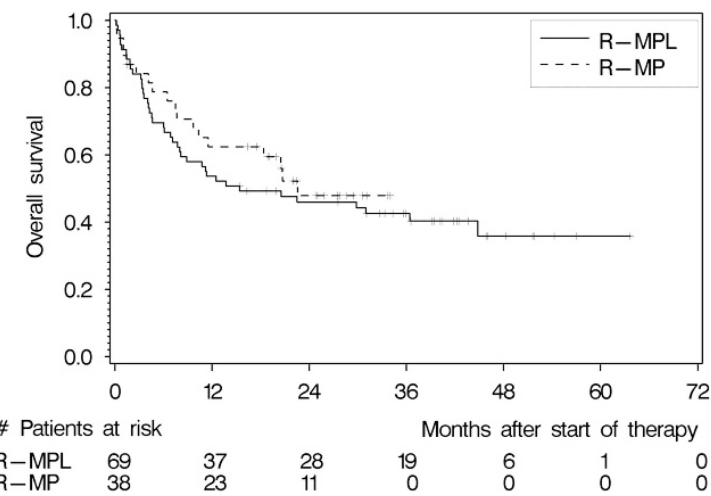

f

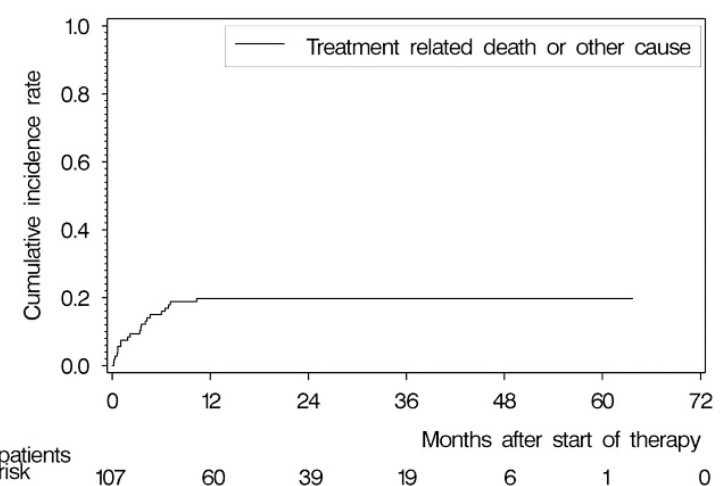

Figure 2. Survival analyses. (a) PFS of the entire cohort, (b) OS of the entire cohort, (c) PFS stratified by R-MPL, (d) OS stratified by R-MPL, (e) cumulative incidence of death owing to lymphoma with other causes of death as competing risk and (f) cumulative incidence of death owing to other causes but lymphoma with lymphoma-associated death as competing risk.

the PRIMAIN study is the largest prospective study specifically designed for elderly PCNSL patients reported so far.

A comparable multicentre single-arm phase II study enrolled 50 patients aged $>60$ years with newly diagnosed PCNSL (EORTC 26925). ${ }^{15}$ Treatment consisted of HD-MTX $\left(1 \mathrm{~g} / \mathrm{m}^{2}\right)$, procarbazine, lomustine, steroids, intrathecal therapy and cytarabine. Regarding best response, $48 \%$ achieved a CR and the median survival was 14.3 months (1-year survival of $52 \%$ ). Similar to our results, myelosuppression was the most common side effect. Another multicentre single-arm study ( $N=89, \geqslant 60$ years) used a combination of HD-MTX-based systemic chemotherapy (HD-AraC, cyclophosphamide, ifosfamide, dexamethasone and vinca-alkaloids) and liposomal cytarabine for intrathecal application. A CR was achieved in $49 \%$ of patients and the 5-year OS was 33\%, which is similar to our findings (33\%); however, it is questionable whether such many different agents and intrathecal treatment provide additional benefit to HD-MTX and HD-AraC. ${ }^{20}$ The first randomized trial ( $N=98$, intergroup ANOCEF-GOELAMS phase II trial) focussing on elderly patients aged $>60$ years compared HD-MTX plus temozolomide and HD-MTX in combination with prednisolone, vincristine and cytarabine for consolidation (MPVA). ${ }^{18}$ Response rates were higher with MPVA ( $82 \%$ vs $71 \%$ ) and also the 2 -year OS ( $58 \%$ vs $39 \%$ ) was in favour of MPVA, although not statistically significant. With all limitations of inter-trial comparison, the bestachieved response rate (by ITT) with MPVA of $79 \%$ was higher compared with our R-MP (57\%); however, the 1-year PFS of $36 \%$ was shorter as compared with R-MP (51\%) and 2-year OS rates were almost identical. These differences highlight the ongoing difficulties in response assessment of PCNSL, which should be centralized if possible in multicentre trials.

Different to our previous studies, ${ }^{8,21}$ we introduced procarbazine maintenance treatment to reduce the risk of relapse. 
Table 4. Multivariable Cox regression model for overall survival

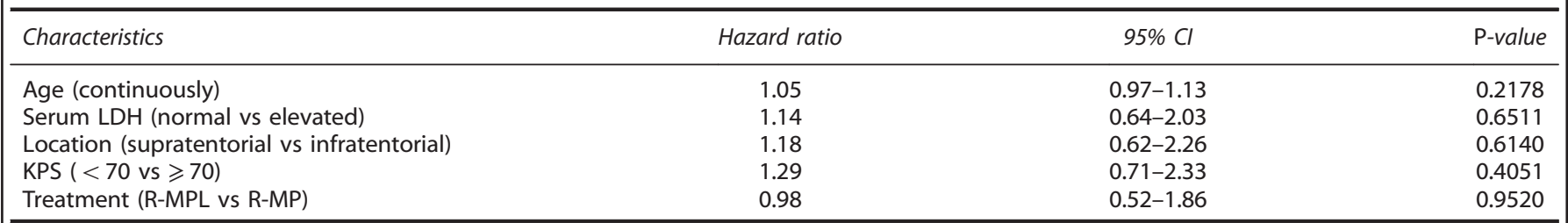

Abbreviations: $\mathrm{Cl}$, confidence interval; KPS, Karnofsky Performance Score; LDH, lactate dehydrogenase; R-MPL, rituximab/methotrexate/procarbazine/ lomustine; R-MP, R-MPL without lomustine.

However, we can only speculate whether this approach was of any success. At least by inter-trial comparison, there was an improvement from $31 \%$ to $44 \%$ in 3 -year OS; future randomized trials are needed to clarify the role of maintenance treatment in PCNSL.

Compared with other prospective trials in elderly patients with PCNSL and systemic diffuse large B-cell lymphoma, the number of treatment-related deaths with R-MPL (8\%) was similar. For example, $5 \%$ of patients in the IELSG 20 trial and $6 \%$ in the IELSG 32 trial died because of treatment-related complications. ${ }^{2,3}$ In the ANOCEF-GOELAMS intergroup trial, 8 (8\%) treatment-related deaths were reported. ${ }^{18}$ Treatment-associated mortality in elderly patients with systemic diffuse large B-cell lymphoma was reported to be $13 \% .^{22}$

Elderly patients constitute the majority of all PCNSL patients and represent a vulnerable population with specific needs. Based on previous work ${ }^{7}$ and the PRIMAIN study, a chemotherapy doublet of HD-MTX and procarbazine together with rituximab (R-MP) is a feasible treatment protocol for elderly PCNSL patients. Around $40 \%$ of patients were not able to finish all three cycles with nine HD-MTX applications. Therefore, in clinical practice, one may consider to start maintenance after two cycles of R-MP if the patient is in CR. The first weeks are crucial to improve the often severely compromised clinical status of these patients. Therefore, future studies need to focus on reducing treatment-associated morbidities to prevent treatment delay and maintenance strategies. This may be achieved by increasing rituximab density in the beginning and introduction of better tolerable novel agents. Given that R-MP seems more feasible in the elderly PCNSL population while still associated with similar efficacy compared with R-MPL, we believe that R-MP should serve as a benchmark for urgently needed comparative trials.

\section{CONFLICT OF INTEREST}

SWK received travel support from Roche. UK reports personal fees from Roche, outside the submitted work. JF reports grants and personal fees from Riemser, grants and personal fees from Novartis, grants and personal fees from Neovii and grants from Medac, outside the submitted work. GH reports grants and personal fees from Roche, outside the submitted work. SK reports non-financial support from Roche, outside the submitted work. The other authors declare no conflict of interest.

\section{ACKNOWLEDGEMENTS}

We thank Dr Elke Valk for her editorial assistance. The trial was sponsored by the Freiburg University Hospital. Additional funding was provided by the Else KrönerFresenius Foundation. The funding bodies had no role in analysing the data or writing the manuscript. Decision for publication was solely made by the academic investigators.

\section{AUTHOR CONTRIBUTIONS}

$\mathrm{Gl}$ and JF designed and supervised the study, recruited patients, interpreted the data and reviewed the manuscript. KF and BK interpreted the data and wrote the manuscript. Glh conducted the statistical analysis. ES, PH, JB, RM, SL, MB, JA,
UK, HHW, SWK, GH, RN, SS, CAS, ML, UM, AM, JP, NF and AR recruited patients and reviewed the manuscript. $\mathrm{CH}$ conducted imaging assessment, $\mathrm{MD}$ conducted neuropathology review and reviewed the manuscript, and HF coordinated the study and reviewed the manuscript.

\section{REFERENCES}

1 Panageas KS, Elkin EB, DeAngelis LM, Ben-Porat L, Abrey LE. Trends in survival from primary central nervous system lymphoma, 1975-1999: a population-based analysis. Cancer 2005; 104: 2466-2472.

2 Ferreri AJM, Reni M, Foppoli M, Martelli M, Pangalis G a, Frezzato M et al. Highdose cytarabine plus high-dose methotrexate versus high-dose methotrexate alone in patients with primary CNS lymphoma: a randomised phase 2 trial. Lancet 2009; 374: 1512-1520.

3 Ferreri AJM, Cwynarski K, Pulczynski E, Ponzoni M, Deckert M, Politi LS et al. Chemoimmunotherapy with methotrexate, cytarabine, thiotepa, and rituximab (MATRix regimen) in patients with primary CNS lymphoma: results of the first randomisation of the International Extranodal Lymphoma Study Group-32 (IELSG32) phase 2 trial. Lancet Haematol 2016; 3: e217-e227.

4 Abrey LE, Yahalom J, DeAngelis LM. Treatment for primary CNS lymphoma: the next step. J Cinical Oncol 2000; 18: 3144-3150.

5 Jahnke K, Korfel A, Martus P, Weller M, Herrlinger U, Schmittel A et al. High-dose methotrexate toxicity in elderly patients with primary central nervous system lymphoma. Ann Oncol 2005; 16: 445-449.

6 Sierra del Rio M, Rousseau A, Soussain C, Ricard D, Hoang-Xuan K. Primary CNS lymphoma in immunocompetent patients. Oncologist 2009; 14: 526-539.

7 Kasenda B, Ferreri AJM, Marturano E, Forst D, Bromberg J, Ghesquieres $\mathrm{H}$ et al. First-line treatment and outcome of elderly patients with primary central nervous system lymphoma (PCNSL)--a systematic review and individual patient data meta-analysis. Ann Oncol 2015; 26: 1305-1313.

8 Fritsch K, Kasenda B, Hader C, Nikkhah G, Prinz M, Haug V et al. Immunochemotherapy with rituximab, methotrexate, procarbazine, and lomustine for primary CNS lymphoma (PCNSL) in the elderly. Ann Oncol 2011; 22: 2080-2085.

9 Abrey LE, Batchelor TT, Ferreri AJM, Gospodarowicz M, Pulczynski EJ, Zucca E et al. Report of an international workshop to standardize baseline evaluation and response criteria for primary CNS lymphoma. J Clin Oncol 2005; 23: 5034-5043.

10 Aaronson NK, Ahmedzai S, Bergman B, Bullinger M, Cull A, Duez NJ et al. The European Organization for Research and Treatment of Cancer QLQ-C30: a quality-of-life instrument for use in international clinical trials in oncology. $J$ Natl Cancer Inst 1993; 85: 365-376.

11 Fleming TR. One-sample multiple testing procedure for phase II clinical trials. Biometrics 1982; 38: 143-151.

12 Gooley TA, Leisenring W, Crowley J, Storer BE. Estimation of failure probabilities in the presence of competing risks: new representations of old estimators. Stat Med 1999; 18: 695-706.

13 Wilson MK, Collyar D, Chingos DT, Friedlander M, Ho TW, Karakasis K et al. Outcomes and endpoints in cancer trials: bridging the divide. Lancet Oncol 2015; 16: e43-e52.

14 Ghesquières H, Ferlay C, Sebban C, Perol D, Bosly A, Casasnovas O et al. Long-term follow-up of an age-adapted C5R protocol followed by radiotherapy in 99 newly diagnosed primary CNS lymphomas: a prospective multicentric phase II study of the Groupe d'Etude des Lymphomes de l'Adulte (GELA). Ann Oncol 2010; 21: 842-850.

15 Hoang-Xuan K, Taillandier L, Chinot O, Soubeyran P, Bogdhan U, Hildebrand J et al. Chemotherapy alone as initial treatment for primary CNS lymphoma in patients older than 60 years: a multicenter phase II study (26952) of the European Organization for Research and Treatment of Cancer Brain Tumor Group. J Clin Oncol 2003; 21: 2726-2731. 
16 Laack NN, Ballman K V, Brown PB, O'Neill BP. Whole-brain radiotherapy and highdose methylprednisolone for elderly patients with primary central nervous system lymphoma: Results of North Central Cancer Treatment Group (NCCTG) 96-73-51. Int J Radiat Oncol Biol Phys 2006; 65: 1429-1439.

17 Olivier G, Clavert A, Lacotte-Thierry L, Gardembas M, Escoffre-Barbe M, Brion A et al. A phase 1 dose escalation study of idarubicin combined with methotrexate, vindesine, and prednisolone for untreated elderly patients with primary central nervous system lymphoma. The GOELAMS LCP 99 trial. Am J Hematol 2014; 89: 1024-1029.

18 Omuro AMP, Chinot O, Taillandier L, Ghesquières H, Soussain C, Delwail V et al. Multicenter randomized phase II trial of methotrexate (MTX) and temozolomide (TMZ) versus MTX, procarbazine, vincristine, and cytarabine for primary CNS lymphoma (PCNSL) in the elderly: an Anocef and Goelams Intergroup study. Lancet Haematol 2015; 2: e251-e259.

19 Pulczynski EJ, Kuittinen O, Erlanson M, Hagberg H, Fosså A, Eriksson $M$ et al. Successful change of treatment strategy in elderly patients with primary central nervous system lymphoma by de-escalating induction and introducing temozolomide maintenance: results from a phase II study by the Nordic Lymphoma Group. Haematologica 2015; 100: 534-540.

20 Schlegel U, Kuhnhenn J, Kowalski T, Steenken S, Pels H, Reiser M et al. Combined systemic polychemotherapy and intrathecal treatment with liposomal cytarabine in patients with primary CNS lymphoma $\geqslant 60$ years: results of a phase II-trial (meeting abstract). Congress of the German Neurology Association (DGN): Hamburg, Germany, 2012.

21 Illerhaus G, Marks R, Müller F, Ihorst G, Feuerhake F, Deckert M et al. High-dose methotrexate combined with procarbazine and CCNU for primary CNS lymphoma in the elderly: results of a prospective pilot and phase II study. Ann Oncol 2009; 20: $319-325$

22 Delarue R, Tilly H, Mounier N, Petrella T, Salles G, Thieblemont C et al. Dose-dense rituximab-CHOP compared with standard rituximab-CHOP in elderly patients with diffuse large B-cell lymphoma (the LNH03-6B study): a randomised phase 3 trial. Lancet Oncol 2013; 14: 525-533.

(i) This work is licensed under a Creative Commons Attributioncc) NonCommercial-NoDerivs 4.0 International License. The images or other third party material in this article are included in the article's Creative Commons license, unless indicated otherwise in the credit line; if the material is not included under the Creative Commons license, users will need to obtain permission from the license holder to reproduce the material. To view a copy of this license, visit http:// creativecommons.org/licenses/by-nc-nd/4.0/

(c) The Author(s) 2017

\section{Supplementary Information accompanies this paper on the Leukemia website (http://www.nature.com/leu)}

